\title{
Hacia la búsqueda de dimensiones más específicas en la medición de la calidad de vida en la esclerosis múltiple
}

\author{
J. Rivera-Navarro å J. Benito-León ${ }^{\text {b }}$ J.M. Morales-González ${ }^{\text {c }}$, grupo GEDMA ${ }^{\text {d }}$
}

\section{SEARCHING FOR MORE SPECIFIC DIMENSIONS FOR THE MEASUREMENT OF QUALITY OF LIFE IN MULTIPLE SCLEROSIS}

\begin{abstract}
Summary. Introduction and objectives. Study of the quality of life in multiple sclerosis $(M S)$ should consider use of an instrument which includes social, psychological, physical and mental aspects. The objective of this study is to show the validated dimensions of the scale of quality of life Functional Assessment of Multiple Sclerosis (FAMS) in the Spanish population, and verify their consistency. Patients and methods. 308 patients with MS (clinically defined or with laboratory support, according to Poser's clinical criteria), referred from 10 Neurology Outpatient Clinics of Hospitals of the Autonomic Community of Madrid. The study was of transversal design, and carried out by direct interview. We used a questionnaire containing the FAMS scale and other variables related to different aspects (clinical, social, employment, psychological and cognitive). Results. The average age was $38.2 \pm 10.5$ years with an average score on the Expanded Disability Status Scale (EDSS) of 3.6 \pm 2.3 . The average scores of the parameters are lower than those obtained with the original version of FAMS; the internal consistency of these dimensions is slightly higher than those of the 'original' FAMS. The parameters 'symptoms' and 'general state of mind' and also the FAMS in general had their internal consistency increased by the inclusion of eight variables which were originally excluded. Conclusion. The FAMS scale is more consistent and reliable for the evaluation of quality of life if we include specific variables of MS which do not form part of the American version of the FAMS. [REV NEUROL 2001; 32: 705-13]
\end{abstract}

Key words. Cognitive deterioration. Depression. Disability. Mobility. Multiple sclerosis. Quality of life.

\section{INTRODUCCIÓN}

La esclerosis múltiple (EM) es una enfermedad con importantes consecuencias psicológicas y sociales para las personas que la padecen, dado que tiene un carácter impredecible en su evolución crónica [1-3]. Dichas consecuencias producen repercusiones en el ámbito poblacional, por ser una enfermedad prevalente en los adultos jóvenes de los países desarrollados [4-16], y limitar los años más productivos de este sector de la población (en el ámbito laboral, familiar y social) [17]. Estas características provocan que los pacientes con EM padezcan no sólo secuelas físicas, sino también problemas sociales y psicológicos [18].

El concepto calidad de vida, relacionado con la salud, se ha utilizado para referirse a las diferentes dimensiones (sociales, psicológicas y clínicas) que se derivan de un suceso patológico. Por lo tanto, actualmente y en el ámbito de las ciencias de la salud, dicho concepto se ha desarrollado con relación a la percepción del individuo sobre las capacidades y limitaciones que una enfermedad conlleva, incluidos los efectos que la entidad y los tratamientos aplicados tienen sobre el estado físico, emocional y sobre su ámbito social [19-23].

Recibido: 10.11.00. Aceptadotras revisión externa sinmodificaciones: 10.12.00.

${ }^{a}$ Unidad de Docencia e Investigación en Medicina Preventiva y Salud Pública. Facultad de Ciencias de la Salud. Universidad Rey Juan Carlos. Alcorcón. ${ }^{b}$ Servicio de Neurología. Hospital General de Móstoles. Móstoles. ${ }^{c}$ Servicio de Investigación. Ministerio de Trabajoy Asuntos Sociales. Madrid, España. ${ }^{d}$ Grupo GEDMA (Grupo de Enfermedades Desmielinizantes de Madrid): M.C. Gutiérrez del Olmo, M.A. Morales (H.U. 12 de Octubre); L. Ayuso, E. Martín-Zurdo (H.U. Príncipe de Asturias); A. Miralles (H.U. La Paz); E. Rodríguez (H. Severo Ochoa); R. Arroyo, C. Martínez-Estefanía (H.U. Clínico San Carlos); C. Ramo (H.U. La Princesa); L. Vela (H. Fundación Alcorcón); A. García-Merino (H.U. Puerta de Hierro) y J. Balseiro (H.U. Getafe).

Correspondencia: Dr. Jesús Rivera Navarro. Unidad de Docencia e Investigación en Medicina Preventiva y Salud Pública. Edificio Departamental. Facultad de Ciencias de la Salud. Universidad Rey Juan Carlos. Avda de Atenas, s/n. E-28922 Alcorcón, Madrid. Fax: +3491488 8848. E-mail: jrivera@cs.urjc.es
En el caso específico de la EM se han realizado diversas investigaciones que analizan la calidad de vida en los pacientes afectados con dicha enfermedad [24-30]; sin embargo, no existe unanimidad en cuanto a los instrumentos de valoración empleados.

Diversos estudios de calidad de vida de la EM han utilizado instrumentos genéricos para su medición, destacando como más relevantes el Short Form-36 (SF-36) [25], el Functional Status Questionaire (FSQ) [26] y el Disability and Impact Profile (DIP) [28]. Sólo recientemente se ha validado un cuestionario que valora la calidad de vida de manera específica en esta entidad, el cuestionario Functional Assessment of Multiple Sclerosis Quality of Life Instrument (FAMS) [31].

Dado el reciente interés en España por incorporar en la evaluación de estos pacientes los aspectos relativos a la calidad de vida $[30,32,33]$, hemos considerado pertinente la realización de un estudio multicéntrico hospitalario en pacientes con EM, para valorar la situación de su calidad de vida. Al utilizar el FAMS como instrumento para la medición de la calidad de vida, el objetivo primordial de este artículo es mostrar el comportamiento que dicha escala ha tenido en la muestra estudiada.

Agradecimientos. Trabajo realizado con la ayuda del laboratorio Schering-Plough (concesión del Premio 1999 de Investigación para la Esclerosis Múltiple) y del laboratorio Schering-España (Ayuda para la Aplicación de los test Mini-Mental State Examination, Hamilton y del reloj, en una muestra de pacientes con esclerosis múltiple). Al Dr. Cella, por darnos facilidades en la aplicación del cuestionario FAMS en nuestra muestra; a Manuel León y Sagrario Cid, por su ayuda en la complementación de los cuestionarios y sus consejos sobre el diseño del estudio; a todos los pacientes que colaboraron en el estudio; a las asociaciones de esclerosis múltiple de Móstoles, Fuenlabrada, Madrid y Getafe por su ayuda y colaboración en todo momento; al Hospital de Móstoles (Gerencia, Comité Ético y Dirección Médica), por proporcionarnos la infraestructura para el estudio y confiar en nosotros; a los hospitales Severo Ochoa, Clínico San Carlos, Getafe, Príncipe de Asturias, 12 de Octubre, Fundación Alcorcón, La Paz, La Princesa y Puerta de Hierro, por facilitarnos el espacio para realizar las entrevistas.

\section{Ó 2001, REVISTA DE NEUROLOGÍA}




\section{PACIENTES Y MÉTODOS}

\section{Población de estudio}

La investigación se realizó con sujetos procedentes de las consultas externas de Neurología de diez hospitales de la Comunidad Autónoma de Madrid: H.U. La Princesa (Área 2), H.U. Príncipe de Asturias (Área 3), H.U. La Paz (Área 5), H.U. Puerta de Hierro (Área 6), H.U. Clínico San Carlos (Área 7), H.G. de Móstoles (Área 8), H. Fundación Alcorcón (Área 8), H.U. Severo Ochoa (Área 9), H.U. Getafe (Área 10), H.U. 12 de Octubre (Área 11).

Los neurólogos, que regularmente atienden a los sujetos con EM de los hospitales anteriormente citados, remitieron aquellos pacientes de su consulta que cumplían los criterios de selección estipulados para el estudio. Se solicitó por escrito la participación de los pacientes seleccionados, explicándoles la finalidad, objetivos y contenidos del estudio, así como garantizándoles la confidencialidad de los datos. Posteriormente, mediante llamada telefónica se concertó la entrevista en el propio hospital de referencia (en 32 casos la entrevista se realizó en el domicilio del paciente o telefónicamente, debido a problemas de movilidad del enfermo).

\section{Criterios de selección}

Los pacientes se seleccionaron a partir de los siguientes criterios de inclusión: a) Sujetos con EM clínicamente definida o con apoyo de laboratorio, según los criterios clínicos de Poser [34], y b) Mayores de 18 años.

Los criterios de exclusión fueron: a) Pacientes institucionalizados en el momento del inicio del estudio; b) Aquellos con enfermedades invalidantes concomitantes (respiratorias, cardiovasculares, gastrointestinales, renales, autoinmunes, hematológicas, endocrinológicas, neurológicas o psiquiátricas) o con cualquier enfermedad crónica grave (cáncer o trastornos psiquiátricos invalidantes) tres meses antes de la inclusión, y c) Aquellos que hubieran participado en cualquier ensayo clínico en los últimos tres meses antes del inicio del estudio.

Los neurólogos nos remitieron a los pacientes, atendiendo a una distribución condicionada por las diferentes formas de presentación de la evolución de la enfermedad en el ámbito poblacional, con el fin de obtener una muestra representativa, según lo manifestado en los diferentes estudios epidemiológicos [4-16].

\section{Diseño del estudio}

El estudio tiene carácter transversal. La investigación se realizó mediante entrevista directa, ya que este método permite la correcta cumplimentación de las preguntas cuando el nivel sociocultural es bajo y, por otra parte, no tiene el sesgo de los encuestados que no responden o contestan parcialmente [35]. Se aplicó un cuestionario para obtener información sobre: a) Datos sociodemográficos (edad, sexo, estado civil, nivel académico); b) La calidad de vida, a través de la utilización de la traducción al castellano del FAMS [31], y las siguientes variables que se estandarizaron previamente [33]: c) La existencia de barreras arquitectónicas en el vecindario y de ayudas técnicas o de terceras personas, en la vida diaria del paciente y en su entorno habitual; d) La influencia de la EM en el trabajo y/o en los estudios, así como las consecuencias del estrés en la propia enfermedad; e) La actitud de la familia ante la aparición de la enfermedad y la repercusión de la misma en la pareja, y f) La existencia de un cuidador principal en la vida del paciente. Al último tercio de los pacientes remitidos se les aplicó también el test de Hamilton (ansiedad y depresión) [36,37], una versión del test Mini-Mental State Examination (MMSE) de Folstein [38] y el test del reloj [39], con la finalidad de realizar una evaluación neuropsicológica de estos enfermos de EM [40].

\section{El instrumento de calidad de vida (FAMS)}

El cuestionario FAMS, validado por Cella et al [31], consta de 59 preguntas (Anexo). De ellas, 44 cuestiones se han estructurado en dimensiones definidas a partir de factores estructurales [31]: movilidad (siete variables), síntomas (siete variables), estado emocional (siete variables), estado de ánimo general (siete variables), actividad mental y fatiga (nueve variables), ambiente familiar y social (siete variables). Las restantes 15 preguntas ('otras preocupaciones') no constituyeron un factor específico en el estudio original, pero contienen cuestiones relacionadas íntimamente con la sintomatología clínica de la EM, tanto por su carácter crónico como por su relación con dicha enfermedad. Aunque todas las preguntas se valoran de 0 a 4 puntos $(0$ nada, 1 un poco, 2 algo, 3 mucho, 4 muchísimo), sólo las anteriores seis subescalas forman una puntuación total en su aplicación. De la dimensión
Tabla I. Datos demográficos, forma clínica de la esclerosis múltiple, EDSS, variables que aluden a la movilidad de los pacientes, así como tests y escalas aplicadas.

\begin{tabular}{|c|c|c|}
\hline & $\begin{array}{c}N .^{\circ} \\
(n=308)\end{array}$ & $\%$ \\
\hline Sexo mujeres & 213 & 69,2 \\
\hline Edad (media + DE) & $38,2 \pm 10,5$ & \\
\hline Estudios primarios completos ${ }^{a}$ & 282 & 91,6 \\
\hline \multicolumn{3}{|c|}{ Forma clínica de la esclerosis múltiple } \\
\hline Remitente-recurrente & 217 & 70,5 \\
\hline Secundariamente progresiva & 63 & 20,5 \\
\hline Primariamente progresiva & 28 & 9,0 \\
\hline \multicolumn{3}{|l|}{ Gravedad EDSS } \\
\hline $0-6$ & 257 & 83,4 \\
\hline $6,5-9$ & 51 & 16,6 \\
\hline $\begin{array}{l}\text { Existencia de barreras } \\
\text { arquitectónicas }\end{array}$ & 137 & 44,5 \\
\hline $\begin{array}{l}\text { Necesidad de algún tipo } \\
\text { de ayudas para andar }\end{array}$ & 129 & 41,9 \\
\hline
\end{tabular}

Ayudas de otras

personas en la vida cotidiana

\begin{tabular}{lcc}
\hline Sí (ayuda continuada) & 62 & 20,1 \\
\hline Sí (ayuda en situaciones concretas) & 111 & 36,1 \\
\hline No & 135 & 43,8 \\
\hline
\end{tabular}

Test MMSE (media \pm DE) 34,2 $\quad 3,3$

Test Hamilton: ansiedad (media \pm DE) $\quad 6,5 \pm 6,0$

Test Hamilton: depresión (media \pm DE) $7,0 \pm 6,9$

Test del reloj: copia (media $\pm \mathrm{DE}) \quad 8,9 \pm 1,9$

Test del reloj: orden (media $\pm \mathrm{DE}$ )

$8,4 \pm 2,4$

DE: desviación estándar; EDSS: Expanded Disability Status Scale; MMSE: Min Mental State Examination de Folstein. ${ }^{a}$ Nos referimos a aquellos pacientes que posean, como mínimo, el título de estudios primarios, así como también incluimos a los que poseen el título de bachillerato o formación profesional, a los diplomados universitarios y a los licenciados universitarios.

'otras preocupaciones' hemos aplicado todas las preguntas -a excepción de una de ellas, por su escasa relevancia en la sintomatología de la enfermedadpara verificar su comportamiento con relación a las anteriores seis subescalas. Todas las respuestas de los sujetos se han codificado en la misma dirección de las puntuaciones, para permitir el sumador de éstas en cada una de las subescalas y su posterior análisis estadístico.

\section{Análisis estadístico}

Se ha llevado a cabo un análisis estadístico descriptivo de las distintas variables; con objeto de establecer la fiabilidad del cuestionario FAMS, se han comparado las puntuaciones medias de dicho test con las de otras variables; se ha hallado el coeficiente alfa de Cronbach para establecer la consistencia interna de las diversas dimensiones del FAMS; se han establecido correlaciones doblemente variadas entre las dimensiones del FAMS y las escalas neuropsicológicas (Hamilton, MMSE y test del reloj), y entre variables excluidas en el FAMS 'original' y las variables de las dimensiones FAMS en las que consideramos pertinente incluir a dichos ítems. 
Tabla II. Influencia de la esclerosis múltiple en el ámbito laboral y académico. Repercusión del estrés en esta enfermedad y la actitud de la familia hacia el paciente.

\begin{tabular}{lcc}
\hline & $\begin{array}{c}\mathrm{N}^{\circ} \\
(\mathrm{n}=308)\end{array}$ & $\%$ \\
\hline Influencia de la EM en el trabajo $^{\text {a }}$ & 66 & 66,7 \\
\hline Influencia de la EM en los estudios $^{\mathrm{b}}$ & 47 & 15,3 \\
\hline Repercusión del estrés en la EM $^{\circ}$ & 239 & 77,6 \\
\hline Actitud de la familia hacia el paciente & & \\
\hline Sobreprotección & 63 & 20,5 \\
\hline Indiferencia & 32 & 10,4 \\
\hline Apoyo, cuando ha sido necesario & 257 & 83,4 \\
\hline Rechazo & 4 & 1,3 \\
\hline Depresión/difícil afrontamiento & 37 & 12,0 \\
\hline Desconocimiento & 29 & 9,4 \\
\hline
\end{tabular}

EM: esclerosis múltiple. a Porcentajes sobre el número parcial $(n=99)$; sólo 99 personas trabajan de forma remunerada (o se encuentran en situación de baja laboral), el resto de los sujetos estudiados tiene pensiones de invalidez (contributiva o no contributiva), pensiones de jubilación, de viudedad, ayudas de bienestar social o subsidio de paro; hay que destacar que el $25,6 \%$ (79 casos) de la serie se dedican a las labores de su hogar. ${ }^{b}$ Las personas que se han visto influidas por la esclerosis múltiple en su vida académica lo han sido en alguna etapa de su vida; no significa que todavía estén estudiando. ${ }^{\circ}$ Las variables que se muestran en el ítem 'actitud de la familia hacia el paciente' no son excluyentes.

\section{RESULTADOS}

La tabla I muestra que el 69,2\% de la serie son mujeres, con una edad media de $38,2 \pm 10,5$ años, y el 60,4\% conviven como pareja estable. Más del $60 \%$ tienen, como mínimo, el título académico de bachillerato y el 32,1\% trabajan actualmente. Respecto a las variables clínicas, el 70,5\% de los pacientes padecían la forma recurrente-remitente, el $20,5 \%$ la forma secundariamente progresiva y el $9 \%$ la primariamente progresiva, siendo la puntuación media de la Expanded Disability Status Scale (EDSS) de 3,6 $\pm 2,3$. Los ítems que aluden a la movilidad de los sujetos muestran que el $56,2 \%$ de los pacientes precisan la colaboración de otras personas en las tareas cotidianas, pero sólo la cuarta parte tiene un cuidador principal que les ayuda en las actividades de la vida diaria. La tabla I muestra también las puntuaciones medias de los tests MMSE, Hamilton (depresión y ansiedad) y del reloj (a la copia y a la orden).

Los datos obtenidos sobre la influencia de la EM en la vida laboral y académica de los pacientes, junto con los que hacen referencia a la repercusión del estrés en la EM y a la actitud de la familia ante la aparición de la enfermedad, se exponen en la tabla II. Más de un tercio de las personas que trabajan de forma remunerada ven afectadas sus obligaciones laborales por la EM; más de las dos terceras partes de la serie considera que el estrés ha influido en la evolución de la EM y más del $80 \%$ de los pacientes se ha sentido apoyado por su familia, con relación a la EM. Es destacable que en un $23,7 \%$ de los sujetos estudiados la enfermedad influyó en su relación de pareja -aunque sólo en un $6,8 \%$ de los casos se produjo ruptura-y que a un $20,1 \%$ de los entrevistados la EM les afectó en la decisión de tener hijos.

\section{Comparación entre el FAMS y otras variables}

Las tablas III y IV muestran el comportamiento de las dimensiones del FAMS en relación con las principales variables estudiadas. Es de destacar que las dimensiones 'síntomas' y 'actividad mental y fatiga' no señalan asociación con la escala EDSS, siendo esta última dimensión la única que presenta relación con el ítem 'la influencia de la EM en la decisión de tener hijos' junto con el 'ambiente familiar y social'; asimismo, sólo la dimensión 'ambiente familiar y social' presenta una fuerte asociación con el apoyo de la familia.

En la tabla V se exponen las principales correlaciones entre las seis dimen-
Tabla III. Comparación de medias entre las dimensiones del cuestionario Functional Assessment of Multiple Sclerosis (FAMS) y otras variables.

\begin{tabular}{lccc}
\hline & Movilidad & Síntomas & $\begin{array}{c}\text { Estado de } \\
\text { ánimo general }\end{array}$ \\
\hline Tipo de esclerosis múltiple & & & \\
\hline RR $(n=217)$ & $8,9 \pm 6,8$ & $8,4 \pm 5,9$ & $7,7 \pm 5,4$ \\
\hline PP y SP $(n=91)$ & $20,1 \pm 6,1^{\text {a }}$ & $9,1 \pm 5,4$ & $14,3 \pm 6,1^{\text {a }}$ \\
\hline EDSS & & & \\
\hline 0-6 $(n=257)$ & $10,2 \pm 7,1$ & $8,6 \pm 5,9$ & $8,5 \pm 5,7$ \\
\hline $6,5-9$ ( $n=51)$ & $20,1 \pm 6,1^{\text {a }}$ & $8,7 \pm 5,3$ & $15,6 \pm 6,1^{\text {a }}$ \\
\hline Influencia en el trabajo & & & \\
\hline Sí $(n=66)$ & $8,8 \pm 6,7$ & $8,5 \pm 5,8$ & $7,7 \pm 4,8$ \\
\hline No $(n=33)$ & $3,6 \pm 3,8^{\text {a }}$ & $4,4 \pm 3,4^{\text {a }}$ & $3,8 \pm 2,3^{\text {a }}$ \\
\hline
\end{tabular}

Influencia en la relación

de pareja

\begin{tabular}{lccc}
\hline Sí $(n=73)$ & $16,7 \pm 7,4$ & $10,4 \pm 6,2$ & $13,3 \pm 6,5$ \\
\hline No $(n=235)$ & $10,8 \pm 8,1^{\text {a }}$ & $8,1 \pm 5,5^{b}$ & $8,5 \pm 5,9^{\text {a }}$ \\
\hline
\end{tabular}

Influencia en la decisión

de tener hijos

\begin{tabular}{llll}
\hline Sí $(n=62)$ & $13,8 \pm 7,7$ & $9,7 \pm 4,9$ & $9,9 \pm 5,6$ \\
\hline No $(n=246)$ & $11,8 \pm 8,4$ & $8,4 \pm 5,9$ & $9,6 \pm 6,6$
\end{tabular}

Existencia de

cuidador principal

\begin{tabular}{llll}
\hline Sí $(n=77)$ & $21,5 \pm 5,8$ & $9,3 \pm 5,6$ & $15,3 \pm 6,1$ \\
\hline No $(n=231)$ & $9,1 \pm 6,5^{a}$ & $8,4 \pm 5,8$ & $7,8 \pm 5,3^{a}$ \\
\hline
\end{tabular}

Repercusión del estrés

en la evolución

\begin{tabular}{llll}
\hline Sí $(n=239)$ & $12,5 \pm 8,2$ & $9,8 \pm 6,4$ & $9,8 \pm 6,4$ \\
\hline No $(n=69)$ & $11,4 \pm 8,5$ & $9,3 \pm 6,4$ & $9,3 \pm 6,4$ \\
\hline
\end{tabular}

Barreras

arquitectónicas

\begin{tabular}{llll}
\hline Sí $(n=137)$ & $17,1 \pm 6,8$ & $10,2 \pm 5,6$ & $12,5 \pm 6,2$ \\
\hline No $(n=171)$ & $8,3 \pm 7,3^{\text {a }}$ & $7,4 \pm 5,6^{\text {a }}$ & $7,4 \pm 5,6^{\text {a }}$ \\
\hline
\end{tabular}

Ayudas para andar

\begin{tabular}{llll}
\hline Sí $(n=129)$ & $19,3 \pm 6,1$ & $9,4 \pm 5,7$ & $13,7 \pm 6,0$ \\
\hline No $(n=179)$ & $7,1 \pm 7,1^{\text {a }}$ & $7,8 \pm 5,7^{\mathrm{b}}$ & $6,7 \pm 4,8^{\mathrm{a}}$ \\
\hline
\end{tabular}

Ayuda de personas

en la vida cotidiana

\begin{tabular}{llll}
\hline Sí $(n=173)$ & $17,0 \pm 6,8$ & $10,4 \pm 5,6$ & $12,4 \pm 6,2$ \\
\hline No $(n=135)$ & $6,0 \pm 5,5^{a}$ & $6,4 \pm 5,2^{b}$ & $6,2 \pm 4,7^{a}$ \\
\hline
\end{tabular}

Apoyo de la familia

\begin{tabular}{llll}
\hline Sí $(n=257)$ & $12,3 \pm 8,3$ & $9,4 \pm 6,1$ & $9,4 \pm 6,1$ \\
\hline No $(n=51)$ & $12,0 \pm 8,5$ & $10,9 \pm 7,5$ & $10,9 \pm 7,5$ \\
\hline
\end{tabular}

EDSS: Expanded Disability Status Scale; RR: recurrente-remitente; SP: secundariamente progresiva; PP: primariamente progresiva. ${ }^{\mathrm{a}} \mathrm{p} £ 0,001 ;{ }^{\mathrm{b}} \mathrm{p} £ 0,01$. 
Tabla IV. Comparación de medias entre las dimensiones del Functional Assessment of Multiple Sclerosis (FAMS) y otras variables.

\begin{tabular}{lccc}
\hline & $\begin{array}{c}\text { Estado } \\
\text { emocional }\end{array}$ & $\begin{array}{c}\text { Actividad mental } \\
\text { /fatiga }\end{array}$ & $\begin{array}{c}\text { Ambiente } \\
\text { familiar } \\
\text { y social }\end{array}$ \\
\hline Tipo de esclerosis múltiple & & & \\
\hline RR $(n=217)$ & $6,5 \pm 5,9$ & $12,9 \pm 8,9$ & $5,4 \pm 4,6$ \\
\hline PP y SP (n=91) & $12,7 \pm 7,1^{\text {a }}$ & $16,4 \pm 8,4^{\text {b }}$ & $7,0 \pm 5,6^{\text {b }}$ \\
\hline EDSS & & & \\
\hline 0-6 $(n=257)$ & $7,2 \pm 6,1$ & $13,6 \pm 8,9$ & $5,4 \pm 4,7$ \\
\hline $6,5-9(n=51)$ & $13,8 \pm 7,9^{\text {a }}$ & $15,8 \pm 9,1$ & $8,4 \pm 5,7^{\text {a }}$ \\
\hline Influencia en el trabajo & & & \\
\hline Sí $(n=66)$ & $6,1 \pm 4,9$ & $12,3 \pm 8,2$ & $4,8 \pm 3,6$ \\
\hline No $(n=33)$ & $1,9 \pm 2,3^{\text {a }}$ & $5,4 \pm 7,2^{\text {a }}$ & $2,3 \pm 3,0^{\text {a }}$ \\
\hline
\end{tabular}

Influencia

en la relación de pareja

\begin{tabular}{llll}
\hline Sí $(n=73)$ & $11,8 \pm 6,6$ & $17,8 \pm 8,7$ & $8,2 \pm 5,4$ \\
\hline No $(n=235)$ & $7,2 \pm 6,5^{a}$ & $12,2 \pm 8,7^{a}$ & $5,1 \pm 4,6^{a}$
\end{tabular}

Influencia en la decisión

de tener hijos

\begin{tabular}{lccc}
\hline Sí $(n=62)$ & $8,1 \pm 5,8$ & $16,6 \pm 7,9$ & $7,3 \pm 4,6$ \\
\hline No $(n=246)$ & $8,3 \pm 7,1$ & $13,3 \pm 9,0^{b}$ & $5,5 \pm 5,0^{b}$ \\
\hline
\end{tabular}

Repercusión del estrés

en la evolución

\begin{tabular}{lccc}
\hline Sí $(n=239)$ & $8,5 \pm 6,8$ & $14,4 \pm 8,9$ & $6,0 \pm 5,1$ \\
\hline No $(n=69)$ & $7,6 \pm 7,2$ & $12,5 \pm 8,8$ & $5,5 \pm 4,6$ \\
\hline
\end{tabular}

Barreras arquitectónicas

\begin{tabular}{cccc}
\hline Sí $(n=137)$ & $11,2 \pm 6,8$ & $17,4 \pm 7,7$ & $7,5 \pm 5,3$ \\
\hline No $(n=171)$ & $5,9 \pm 6,0^{a}$ & $11,2 \pm 8,9^{\text {a }}$ & $4,5 \pm 3,7^{\text {a }}$ \\
\hline Ayuda para andar & & & \\
\hline Sí $(n=129)$ & $12,3 \pm 6,8$ & $17,1 \pm 8,0$ & $7,5 \pm 5,3$ \\
\hline No $(n=179)$ & $5,4 \pm 5,3^{\text {a }}$ & $11,7 \pm 8,9^{\text {a }}$ & $4,9 \pm 4,5^{\text {a }}$ \\
\hline
\end{tabular}

Ayuda de personas

en la vida cotidiana

\begin{tabular}{cccc}
\hline Sí $(n=173)$ & $11,2 \pm 6,8$ & $17,7 \pm 7,9$ & $7,2 \pm 5,4$ \\
\hline No $(n=135)$ & $4,5 \pm 4,9^{a}$ & $9,2 \pm 7,9^{a}$ & $4,1 \pm 3,6^{a}$ \\
\hline Apoyo de la familia & & & \\
\hline Sí $(n=257)$ & $7,9 \pm 6,9$ & $13,9 \pm 9,0$ & $5,2 \pm 4,3$ \\
\hline No $(n=51)$ & $10,2 \pm 8,0^{\circ}$ & $14,2 \pm 8,6$ & $9,2 \pm 6,5^{a}$ \\
\hline
\end{tabular}

EDSS: Expanded Disability Status Scale; RR: recurrente-remitente; SP: secundariamente progresiva; $\mathrm{PP}$ : primariamente progresiva. ${ }^{a} \mathrm{p} £ 0,001 ;{ }^{\mathrm{b}} \mathrm{p} £ 0,01 ;{ }^{\circ} \mathrm{p} £ 0,05$

siones delFAMS y los tests MMSE y del reloj (a la orden y a la copia) y la escala de Hamilton (ansiedad y depresión). Destacamos la inexistencia de asociaciones significativas entre el MMSE y la mayoría de las dimensiones, excepto las subescalas de 'estado de ánimo general' y 'estado emocional'. Todas las di-
Tabla V. Principales correlaciones de las dimensiones del Functional Assessment of Multiple Sclerosis con la escala de Hamilton (depresión y ansiedad), el Mini Mental State Examination y test del reloj.

\begin{tabular}{lcccccc}
\hline & $M$ & $S$ & $S G$ & $E E$ & $P / F$ & $A F S$ \\
\hline & $C C$ & $C C$ & $C C$ & $C C$ & $C C$ & $C C$ \\
\hline MMSE & $-0,151^{1}$ & $-0,038$ & $-0,193^{c}$ & $-0,191^{c}$ & $-0,161$ & $-0,151$ \\
\hline H. an. & $0,488^{a}$ & $0,527^{a}$ & $0,469^{a}$ & $0,522^{a}$ & $0,526^{a}$ & $0,469^{a}$ \\
\hline H. de. & $0,598^{a}$ & $0,469^{a}$ & $0,542^{a}$ & $0,563^{a}$ & $0,502^{a}$ & $0,334^{a}$ \\
\hline O. re. & $-0,240^{b}$ & $-0,086$ & $-0,369^{a}$ & $-0,366^{a}$ & $-0,157$ & $-0,240^{b}$ \\
\hline C. re. & $-0,395^{a}$ & $-0,144$ & $-0,407^{a}$ & $-0,371^{a}$ & $-0,205^{c}$ & $-0,227^{c}$ \\
\hline
\end{tabular}

M: movilidad; S: síntomas; SG: estado de ánimo general; EE: estado emocional P/F: pensamiento/fatiga; AFS: ambiente familiar y social; CC: coeficiente de correlación: MMSE: Mini Mental State Examination; $H$. an.: puntuación de la escala de Hamilton (ansiedad); H. de.: puntuación de la escala de Hamilton (depresión); O. r.: puntuación del test del reloj (a la orden); C. re.: puntuación test de reloj (a la copia). ${ }^{a} p £ 0,001 ;{ }^{b} p £ 0,01 ;{ }^{c} p £ 0,05$.

Tabla VI. Principales correlaciones de algunas de las variables excluidas del Functional Assessment of Multiple Sclerosis 'original' con los ítems de las dimensiones 'síntomas' y 'estado de ánimo general'.

Sínt. Náusea Dolor Enferm. Débil Articu. D. cabe. D. mus.

\begin{tabular}{lccccccc}
\hline & $C C$ & $C C$ & $C C$ & $C C$ & $C C$ & $C C$ & $C C$ \\
\hline Efecto & 0,956 & $0,205^{a}$ & $0,199^{c}$ & $0,199^{a}$ & 0,020 & $0,163^{b}$ & $0,18^{c}$ \\
\hline R. sex. & $0,180^{a}$ & $0,225^{b}$ & $0,302^{a}$ & $0,302^{a}$ & $0,244^{a}$ & 0,053 & $0,22^{a}$ \\
\hline Duerm. & $0,265^{a}$ & $0,306^{a}$ & $0,133^{b}$ & $0,250^{a}$ & $0,272^{a}$ & $0,301^{a}$ & $0,20^{a}$ \\
\hline Calor & 0,097 & $0,224^{a}$ & $0,192^{a}$ & $0,335^{a}$ & 0,103 & $0,186^{a}$ & $0,11^{c}$ \\
\hline C. orin. & 0,071 & $0,197^{a}$ & $0,261^{a}$ & $0,261^{a}$ & $0,185^{a}$ & 0,173 & $0,22^{a}$ \\
\hline Orin. & 0,065 & $0,186^{a}$ & $0,165^{b}$ & $0,177^{b}$ & $0,156^{b}$ & $0,155^{b}$ & $0,14^{b}$ \\
\hline Espas. & $0,143^{b}$ & $0,232^{a}$ & $0,256^{a}$ & $0,279^{a}$ & $0,269^{a}$ & $0,135^{b}$ & $0,36^{a}$ \\
\hline SG & S. trab. & Acep. & D. pasa. & Calidad & F. cond. & V. sent. & Motiv. \\
\hline & CC & CC & CC & CC & CC & CC & CC \\
\hline S. afr. & $0,420^{a}$ & $0,361^{a}$ & $0,530^{a}$ & $0,494^{a}$ & $0,455^{a}$ & $0,514^{a}$ & $0,48^{a}$ \\
\hline
\end{tabular}

Sínt.: dimensión de la FAMS 'síntomas'; Náusea: 'Tiene náuseas' (variable de 'síntomas'): Dolor: 'Tiene dolor' (variable de 'síntomas'): Enferm.: 'Se siente enfermo/a' (variable de 'síntomas'); Débil: 'Siente debilidad en todo el cuerpo' (variable de 'síntomas'); Articu.: 'Tiene dolor en sus articulaciones' (variable de 'síntomas'); D. cabe.: 'Se siente molesto/a con los dolores de cabeza' (variable de 'síntomas'); D. mus.: 'Se siente molesto/a con los dolores musculares' (variables de 'síntomas'). Efecto: 'Le molestan los efectos secundarios del tratamiento' (variable de 'otras preocupaciones'): R. sex.:' ¿Está satisfecho con su vida sexual? (variable de 'otras preocupaciones'); Duerm.: 'Duerme bien' (variable de 'otras preocupaciones'); Calor: 'Siente que empeoran los síntomas de la enfermedad con el calor' (variable de 'otras preocupaciones'); C. Orin.: 'Tiene dificultad para controlar la orina' (variable de 'otras preocupaciones'); Orin.: 'Orina con más frecuencia que normalmente' (variable de 'otras preocupaciones'); Espas.: 'Se siente molesto/a con los espasmos musculares' (variable de 'otras preocupaciones'): S.G.: estado de ánimo general; S. trab.: 'Le satisface su trabajo (incluido el trabajo en casa)' (variable de 'estado de ánimo general'); Acep.: 'Ha aceptado su enfermedad' (variable de 'estado de ánimo general'); D. pasa.: 'Disfruta con sus pasatiempos de siempre' (variable de 'estado de ánimo general'); Calidad: 'Está satisfecho con su vida (calidad de vida actual)' (variable de 'estado de ánimo general'); F. cond.: 'Está frustrado por su condición (por su estado de salud)' (variable de 'estado de ánimo general'); V. sent.: 'Siente que su vida tiene sentido' (variable de 'estado de ánimo general'); Motiv.: 'Se siente motivado para hacer cosas' (variable de 'estado de ánimo general'); S. afr.: 'Está satisfecho de la manera en que usted está afrontando la enfermedad' (variable de 'otras preocupaciones'). CC: coeficiente de correlación; ${ }^{a} p £ 0,001 ;{ }^{b} p £ 0,01 ;{ }^{c} p £ 0,05$. 
Tabla VII. Dimensiones del Functional Assessment of Multiple Sclerosis y puntuaciones, incluida la consistencia interna.

\begin{tabular}{|c|c|c|c|}
\hline & Intervalo & $\begin{array}{l}\text { Media } \pm D E \\
\quad(n=308)\end{array}$ & C. alfa \\
\hline Movilidad (7 variables) & $0-28$ & $12,2 \pm 8,3$ & 0,90 \\
\hline Síntomas (7 variables) & $0-56$ & $22,3 \pm 12,5$ & 0,94 \\
\hline Síntomas (14 variables) ${ }^{a}$ & $0-28$ & $8,6 \pm 5,8$ & 0,77 \\
\hline $\begin{array}{l}\text { Estado emocional } \\
\text { (7 variables) }\end{array}$ & $0-28$ & $8,3 \pm 6,9$ & 0,85 \\
\hline $\begin{array}{l}\text { Estado de ánimo general } \\
\text { (7 variables) }\end{array}$ & $0-28$ & $8,8 \pm 7,0$ & 0,84 \\
\hline 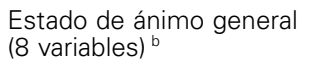 & $0-32$ & $9,7 \pm 7,9$ & 0,86 \\
\hline $\begin{array}{l}\text { Pensamiento y fatiga } \\
\text { (9 variables) }\end{array}$ & $0-36$ & $14,0 \pm 8,9$ & 0,89 \\
\hline $\begin{array}{l}\text { Familia/bienestar social } \\
\text { (7 variables) }\end{array}$ & $0-28$ & $5,9 \pm 5,0$ & 0,73 \\
\hline FAMS total (44 variables) & $0-176$ & $57,8 \pm 41,9$ & 0,94 \\
\hline FAMS total (52 variables) ${ }^{\circ}$ & $0-208$ & $68,8 \pm 47$ & 0,95 \\
\hline
\end{tabular}

FAMS: Functional Assessment of Multiple Sclerosis; DE: desviación estándar: C. alfa: coeficiente alfa de Cronbach. ${ }^{a}$ Se incluyen también algunas de las variables que Cella et al excluyeron del cuestionario FAMS y que se incluían en la dimensión 'otras preocupaciones'; estos ítems son: 'Le molestan los efectos secundarios del tratamiento', '¿Está satisfecho con su vida sexual?', '¿Duerme bien?', 'Siente que empeoran los síntomas con el calor', 'Tiene dificultad para controlar la orina', 'Orina con más frecuencia que normalmente', 'Se siente molesto con los espasmos musculares'. 'Se incluye una de las variables que Cella et al [31] excluyeron del FAMS y que se incluía en la dimensión 'otras preocupaciones'; este ítem es: '¿Está satisfecho de cómo está afrontando la enfermedad?. ' Se calcula la media \pm DE y el coeficiente alfa de Cronbach para la puntuación total del FAMS, teniendo en cuenta las ocho variables antes citadas.

mensiones revelan correlaciones muy significativas con la escala de Hamilton (en sus variantes de depresión y ansiedad). Es importante señalar las correlaciones existentes entre el test del reloj (especialmente la modalidad 'a la copia') y todas las subescalas (excepto con la dimensión 'síntomas').

\section{Consistencia interna de las dimensiones del FAMS}

El empleo de algunas de las variables excluidas en el FAMS 'original' [31] se justifica en las tablas VI y VII. En la primera se establecen correlaciones doblemente variadas entre las variables 'espurias' y los ítems de las dimensiones 'síntomas' y 'estado de ánimo general' -en dichas dimensiones se ubican estas variables-. En la segunda se indican las puntuaciones y consistencias de las seis subescalas del FAMS. Las puntuaciones medias apuntan valores no excesivamente altos (intervalo de 5,9 a 14,0), y los coeficientes alfa de Cronbach presentan valores por encima de 0,7 .

En la tabla VII también se muestran los resultados obtenidos al incorporar en las subescalas 'síntomas' y 'estado de ánimo general' aquellas preguntas incluidas en el apartado 'otras preocupaciones', que se relacionan significativamente con estas dos dimensiones. La consistencia interna de dichas subescalas 'modificadas' son más altas que las dimensiones establecidas originariamente, de forma que el FAMS total, incluyendo 52 variables, mantiene una fiabilidad total semejante al FAMS de 44 variables.

\section{DISCUSIÓN}

El presente estudio está constituido por una muestra de sujetos representativa de la EM. Dichos pacientes tienen unas características sociodemográficas y clínicas semejantes a lo descrito en los diversos estudios epidemiológicos [16,25,30,41]. Por ello, los resultados obtenidos sobre la situación de la calidad de vida de estos pacientes son extrapolables a la población afectada por esta enfermedad.

Por una parte, los aspectos de la calidad de vida de nuestro estudio indican divergencias con investigaciones realizadas en nuestro ámbito, las cuales han utilizado muestras clínicas no representativas en el ámbito poblacional [26,33]. Dichas diferencias pueden observarse claramente en variables como la percepción de barreras arquitectónicas en la vida cotidiana y la necesidad de ayuda técnica para andar, que en nuestro estudio alcanzan porcentajes del 40-45\%. Por otra parte, un estudio recientemente realizado en el Área Sanitaria 1 de Madrid, con una muestra ambulatoria, presentó resultados similares a los nuestros con relación a la necesidad de ayuda física [30]. La ayuda de terceras personas en diversas situaciones de la vida cotidiana, ya sea ésta continuada o en momentos concretos, se produce en más de la mitad de nuestra serie, coincidiendo con lo expresado en diversas publicaciones nacionales e internacionales [17,25,26,28,30,42].

El estudio del impacto de la EM en el ámbito laboral y académico se ha reflejado con relativa profusión en el medio científico y, aunque es difícil comparar nuestros resultados con los hallados en otras investigaciones, debido a las variaciones producidas por el tipo de pacientes analizados y el tiempo de seguimiento, sí hay unanimidad en todos los estudios en la relación de esta enfermedad con la problemática laboral y académica $[29,30,32,42,43]$.

La asociación entre el estrés, la depresión y la EM también ha dado lugar a numerosos trabajos [18,44-46]. En nuestro estudio, más de las dos terceras partes de los sujetos manifiestan que el estrés tuvo repercusión en el desarrollo de la enfermedad; sin embargo, el análisis del test de Hamilton revela puntuaciones medias por debajo del nivel de ansiedad y depresión, sin soslayar la gran variabilidad existente entre los pacientes en este sentido.

La repercusión de la EM en las relaciones maritales se ha reflejado en nuestro estudio, donde los resultados se acercan a los de algunas investigaciones de carácter similar [30,47]. El apoyo de la familia ha sido confirmado por la mayoría de los sujetos entrevistados, variable que también han tenido en cuenta otros autores [17].

Siguiendo las recomendaciones del estudio original de Cella et al [31], hemos considerado pertinente la aplicación del FAMS en su totalidad (58 variables, ya que se eliminó una de ellas, como ya se ha explicado en el apartado de Pacientes y Métodos), con seis dimensiones definidas y una séptima área con diversos ítems específicos de la EM, para poder verificar el comportamiento de una adaptación al castellano de esta escala, en la medición de la calidad de vida, en una muestra con características clínicas y sociodemográficas similares a la población afectada por esta enfermedad.

Los resultados obtenidos en este estudio indican que las seis subescalas del FAMS están relacionadas con las principales variables estudiadas en la calidad de vida de estos pacientes.

La asociación de las dimensiones 'movilidad', 'estado de ánimo general', 'estado emocional' y 'ambiente familiar y social' con la forma de evolución de la EM y la incapacidad (medida por la escala EDSS) corrobora lo observado en otros estudios, que apuntan a que la incapacidad física condiciona las relaciones sociales y la dependencia familiar [26-30,32,42]. La relación entre la EM $\mathrm{y}$, por lo tanto, la calidad de vida de los que la padecen y la depresión ha sido estudiada detalladamente por diversos autores $[18,44-46,48]$. No obstante, no podemos obviar que se ha planteado como variable intermedia en esta relación el grado de depresión 
de los pacientes [45,48]. En nuestro estudio las seis subescalas están fuertemente relacionadas con el grado de depresión y ansiedad, medido por el test de Hamilton.

El impacto de la EM en el trabajo y en la vida marital es el argumento que justifica la relación entre las seis dimensiones y los ítems que hacen referencia a ambos temas. Dicha influencia se ha explicado y analizado en diversos trabajos [17,29,21,32]. Además, la asociación entre cada una de las seis dimensiones y la necesidad de ayudas personales y técnicas, junto con la percepción de barreras arquitectónicas en el vecindario, son determinantes en la calidad de vida del sujeto [26].

El deterioro cognitivo en la EM y su influencia en la calidad de vida se ha analizado también en nuestro estudio. El MMSE de Folstein tan sólo se asocia con la dimensión 'estado de ánimo general' y 'estado emocional', e indica sobre todo la existencia de problemas de atención y de fatiga, ya que en nuestra muestra los sujetos no padecen un deterioro cognitivo grave y esta entidad no cursa, en general, con demencia, como otras enfermedades neurológicas crónicas [49]. En cambio, el test del reloj, en ambas versiones (a la orden y a la copia), correlaciona con todas las dimensiones, excepto con 'síntomas', debido probablemente a que la EM puede presentarse con trastornos cognitivos relacionados con la memoria reciente, la atención mantenida y la percepción [50,51]. La relación entre el deterioro cognitivo, la depresión y la EM ya se ha referenciado en otros estudios, aunque se han utilizado instrumentos diferentes al MMSE y al test del reloj [52,53].

Las puntuaciones medias de las seis subescalas obtenidas en nuestro estudio son inferiores y con mayor desviación estándar a las mostradas por el estudio original [31]. Dichas diferencias responden a la heterogeneidad, en cuanto a la discapacidad, tipo de EM y afectación de la misma en nuestra serie. Cella et al [31] incluyen en su estudio sujetos con mayor gravedad en su enfermedad y, por lo tanto, con peor calidad de vida.

La consistencia interna de cada una de las dimensiones y del conjunto de ellas resultó similar a la del FAMS original, reflejan- do la homogeneidad de las preguntas incluidas en cada dimensión y de la escala total, con un alfa de Cronbach de 0,94.

En este estudio hemos utilizado las preguntas que en el estudio original se excluyeron de las dimensiones de calidad de vida, aunque agrupadas en una 'pseudodimensión' llamada 'otras preocupaciones' [31]. Debido a que en nuestro estudio hemos utilizado el FAMS como instrumento central para medir la calidad de vida, hemos analizado de forma previa nuestra base de datos para verificar el comportamiento de ésta, observando que más de la mitad de las preguntas están muy relacionadas con dos de las dimensiones ya establecidas en la versión original: 'síntomas' y 'estado de ánimo general'. El hallazgo en este trabajo fue observar cómo algunos de dichos ítems son válidos para reforzar dos de las dimensiones ya contrastadas y mejorar la evaluación de la calidad de vida de los pacientes con EM.

La dimensión 'síntomas' pasaría a estar conformada por 14 variables al añadirse siete preguntas, y la subescala 'estado de ánimo general' se convierte en una dimensión con ocho variables, ya que se añade un ítem. La consistencia interna de estas dos nuevas subescalas es mayor que las originalmente planteadas, de forma que elFAMS con 52 variables -incluidas las ocho variables antes excluidas- muestra una consistencia interna similar a la versión original de 44 ítems. Todas las preguntas están muy vinvuladas con dos dimensiones ya establecidas en la versión original: 'síntomas' y 'estado de ánimo general'.

Los análisis realizados para la exploración de las variables del FAMS y el estudio del comportamiento de ésta (fase preliminar de cualquier investigación epidemiológica, para determinar un plan de análisis definitivo) ha mostrado la capacidad del FAMS para valorar la calidad de vida de los pacientes con EM. Los análisis de fiabilidad interna de la escala sugieren la posibilidad de incorporar aquellos ítems más específicos con relación a la EM, más aún cuando éstos tienen una relevancia clínica para esta entidad. Posteriores estudios de validación de esta escala deberán contemplar la validez y fiabilidad de las diferentes subescalas, con el fin de dotar a dicho instrumento de la adaptación específica a esta enfermedad.

\section{BIBLIOGRAFÍA}

1. Patterson MB, Fliart R. Multiple sclerosis: understanding the psychologic implications. Gen Hosp Psychiatry 1985; 8: 234-8.

2. Murray TJ. The psychosocial aspects of multiple sclerosis. Neurol Clin 1995; 13: 197-223.

3. Lectenberg R. Multiple sclerosis fact book. Philadelphia: FA Davis; 1988.

4. Kurtze JF. Epidemiology of multiple sclerosis. In Vinken PJ, Bruyn GW, Klawans HL, eds. Handbook of clinical neurology. Demyelinating diseases. Amsterdam: Elsevier; 1985. p. 259-87.

5. Swingler RJ, Kirk PF, Darke C, Compston DAS. HLA and multiple sclerosis in South-East Wales. J Neurol Neurosurg Psychiatry 1987; 50: $1153-5$.

6. Firnhaber W, Lauer K. Multiple sclerosis in Europe. An epidemiological update. Alsbach-Bergstrasse: Leuchtturn-Verlag/LTV Press; 1994.

7. Granieri E, Casetta M, Tola R. Epidemiology of multiple sclerosis in Italy and in Southern Europe. Acta Neurol Scand 1996; 161: 60-70.

8. Rosati G, Aiello I, Pirastru MI, Mannu L, Sanna G, Sau GF, et al. Epidemiology of multiple sclerosis in Northwestern Sardinia: further evidence for higher frequency in Sardinians compared to other Italians. Neuroepidemiology 1996; 15: 10-9.

9. Dean G, Aksoy H, Akalin T, Middleton L, Kyriallis K. Multiple sclerosis in the Turkish- and Greek-speaking communities of $\mathrm{Cy}$ prus. A United Nations (UNHCR) bicommunal project. J Neurol Sci 1997; 145: 163-8.

10. Savettieri G, Salemi G, Ragonese P, Arindon P, Scola G, Randisi G. Prevalence and incidence of multiple sclerosis in the city of Montreale, Italy. J Neurol 1998; 245: 40-3.

11. McDonell GV, Hawkins SA. An epidemiologic study of multiple sclerosis in Northern Ireland. Neurology 1998; 50: 423-8.

12. Matías-Guiu J, Bolumar F, Martín R, Insa R, Casquero P, Moltó JM, et al. Multiple sclerosis in Spain: an epidemiological study of the Alcoy health region, Valencia. Acta Neurol Scand 1990; 81: $479-83$.

13. Fernández O, Luque G, San Román C, Bravo M, Dean G. The prevalence of multiple sclerosis in the sanitary district of Vélez-Málaga, central Spain. Neurology 1994; 44: 425-9.

14. Uria DF, Calatayud MT, Virgala P, Díaz A, Chamizo C, Dean G. Multiple sclerosis in Gijon health district, Asturias, Northern Spain. Acta Neurol Scand 1997; 96: 375-9.

15. Modrego-Pardo PJ, Pina Latorre MA, López A, Errea JM. Prevalence of multiple sclerosis in the province of Teruel, Spain. J Neurol 1997; 244: 182-5.

16. Benito-León J, Martín E, Vela L, Villar ME, Felgueroso B, Marrero C, et al. Multiple sclerosis in Móstoles, central Spain. Acta Neurol Scand 1998; 98: 238-42.

17. Stenager E, Stenager EN, Knudsen L, Jensen K. Multiple sclerosis: the impact on family and social life. Acta Psychiatr Belg 1994; 94 : 165-74.

18. Mohr DC, Dick LP, Russo D, Pinn J, Boudewyn AC, Likosky W, et al. The psychosocial impact of multiple sclerosis: exploring the patient's perspective. Health Psychol 1999; 18: 376-82.

19. Testa MA, Simonson DC. Assessment of quality of life outcomes. N Engl J Med 1996; 13: 835-40.

20. Buttler RN. Quality of life: can it be an endpoint? How can it be measured? Am J Clin Nutr 1992; 55: 1267-70.

21. McFarlane AH, Norma GR, Streiner DL, Roy RG. Characteristics and correlates of effective and ineffective social supports. J Psychosom Res 1984; 28: 501-10.

22. Linn MW, Klett CJ, Caffey EM. Foster home characteristics and psy- 
Anexo. Ponderación de la calidad de vida en la esclerosis múltiple mediante la complementación del cuestionario adaptado al castellano Functional Assessment of Multiple Sclerosis (59 variables). Presentación al sujeto entrevistado: 'A continuación, se le presentará una lista de afirmaciones sobre situaciones muy comunes en personas con su misma enfermedad. Dependiendo de lo cierto que haya sido para usted cada afirmación durante los últimos siete días, por favor, indíquenos sólo uno de los números que aparecen en cada línea'.

\begin{tabular}{|c|c|c|c|c|c|}
\hline Escala & 0. Nada & 1. Un poco & 2. Algo & 3. Mucho & 4. Muchísimo \\
\hline \multicolumn{6}{|l|}{ Movilidad } \\
\hline $\begin{array}{l}\text { 1. A causa de su estado físico, tiene problemas para atender } \\
\text { las necesidades de su familia }\end{array}$ & 0 & 1 & 2 & 3 & 4 \\
\hline 2. Puede trabajar (incluido el trabajo en casa) & 0 & 1 & 2 & 3 & 4 \\
\hline 3. Tiene dificultad para caminar & 0 & 1 & 2 & 3 & 4 \\
\hline 4. Ha limitado su actividad social a causa de su estado de salud & 0 & 1 & 2 & 3 & 4 \\
\hline 5. Tiene fuerza en las piernas & 0 & 1 & 2 & 3 & 4 \\
\hline 6. Tiene dificultad para desplazarse a sitios públicos & 0 & 1 & 2 & 3 & 4 \\
\hline 7. Tiene que organizarse en función de su estado de salud & 0 & 1 & 2 & 3 & 4 \\
\hline \multicolumn{6}{|l|}{ Síntomas } \\
\hline 8. Tiene náuseas & 0 & 1 & 2 & 3 & 4 \\
\hline 9. Tiene dolor & 0 & 1 & 2 & 3 & 4 \\
\hline 10. Se siente enfermo/a & 0 & 1 & 2 & 3 & 4 \\
\hline 11. Siente debilidad en todo el cuerpo & 0 & 1 & 2 & 3 & 4 \\
\hline 12. Tiene dolor en sus articulaciones & 0 & 1 & 2 & 3 & 4 \\
\hline 13. Se siente molesto/a con los dolores de cabeza & 0 & 1 & 2 & 3 & 4 \\
\hline 14. Se siente molesto/a con los dolores musculares & 0 & 1 & 2 & 3 & 4 \\
\hline \multicolumn{6}{|l|}{ Estado emocional } \\
\hline 15. Se siente triste & 0 & 1 & 2 & 3 & 4 \\
\hline 16. Está perdiendo la esperanza en la lucha contra su enfermedad & 0 & 1 & 2 & 3 & 4 \\
\hline 17. Puedo disfrutar de la vida & 0 & 1 & 2 & 3 & 4 \\
\hline 18. Se siente prisionero por su estado de salud & 0 & 1 & 2 & 3 & 4 \\
\hline 19. Se siente deprimido por su estado de salud & 0 & 1 & 2 & 3 & 4 \\
\hline 20. Se siente inútil & 0 & 1 & 2 & 3 & 4 \\
\hline 21. Se siente agobiado por su estado de salud & 0 & 1 & 2 & 3 & 4 \\
\hline \multicolumn{6}{|l|}{ Estado de ánimo general } \\
\hline 22. Le satisface su trabajo (incluido el trabajo en casa) & 0 & 1 & 2 & 3 & 4 \\
\hline 23. Ha aceptado su enfermedad & 0 & 1 & 2 & 3 & 4 \\
\hline 24. Disfruta con sus pasatiempos de siempre & 0 & 1 & 2 & 3 & 4 \\
\hline 25. Está satisfecho con su vida (calidad de vida) actual & 0 & 1 & 2 & 3 & 4 \\
\hline 26. Está frustrado por su condición (por su estado de salud) & 0 & 1 & 2 & 3 & 4 \\
\hline 27. Siente que su vida tiene sentido & 0 & 1 & 2 & 3 & 4 \\
\hline 28. Se siente motivado para hacer cosas & 0 & 1 & 2 & 3 & 4 \\
\hline \multicolumn{6}{|l|}{ Actividad mental y fatiga } \\
\hline 29. Le falta energía & 0 & 1 & 2 & 3 & 4 \\
\hline 30. Se siente cansado & 0 & 1 & 2 & 3 & 4 \\
\hline 31. Tiene dificultad para comenzar las cosas porque está cansado & 0 & 1 & 2 & 3 & 4 \\
\hline 32. Tiene dificultad para terminar las cosas porque está cansado & 0 & 1 & 2 & 3 & 4 \\
\hline
\end{tabular}




\section{J. RIVERA-NAVARRO, ET AL}

Anexo (continuación). Ponderación de la calidad de vida en la esclerosis múltiple mediante la complementación del cuestionario adaptado al castellano Functional Assessment of Multiple Sclerosis ( 59 variables). Presentación al sujeto entrevistado: 'A continuación, se le presentará una lista de afirmaciones sobre situaciones muy comunes en personas con su misma enfermedad. Dependiendo de lo cierto que haya sido para usted cada afirmación durante los últimos siete días, por favor, indíquenos sólo uno de los números que aparecen en cada línea'.

\begin{tabular}{|c|c|c|c|c|c|}
\hline Escala & 0. Nada & 1. Un poco & 2. Algo & 3. Mucho & 4. Muchísimo \\
\hline \multicolumn{6}{|l|}{ Actividad mental y fatiga } \\
\hline 33. Necesita descansar durante el día & 0 & 1 & 2 & 3 & 4 \\
\hline 34. Tiene dificultad para recordar las cosas & 0 & 1 & 2 & 3 & 4 \\
\hline 35. Tiene dificultad para concentrarse & 0 & 1 & 2 & 3 & 4 \\
\hline 36. Su actividad mental es más lenta que antes & 0 & 1 & 2 & 3 & 4 \\
\hline $\begin{array}{l}\text { 37. Tiene dificultad para aprender cosas nuevas } \\
\text { o recordar instrucciones }\end{array}$ & 0 & 1 & 2 & 3 & 4 \\
\hline \multicolumn{6}{|l|}{ Ambiente familiar y social } \\
\hline 38. Se siente cercano/a a sus amistades & 0 & 1 & 2 & 3 & 4 \\
\hline 39. Tiene el apoyo emocional de su familia & 0 & 1 & 2 & 3 & 4 \\
\hline 40. Tiene el apoyo de sus amistades & 0 & 1 & 2 & 3 & 4 \\
\hline 41. Su familia ha aceptado su enfermedad & 0 & 1 & 2 & 3 & 4 \\
\hline $\begin{array}{l}\text { 42. Se siento satisfecho/a con la manera en que se comunica } \\
\text { con su familia acerca de su enfermedad }\end{array}$ & 0 & 1 & 2 & 3 & 4 \\
\hline 43. A su familia le cuesta entender que su estado empeore & 0 & 1 & 2 & 3 & 4 \\
\hline 44. Siente que le marginan & 0 & 1 & 2 & 3 & 4 \\
\hline \multicolumn{6}{|l|}{ Otras preocupaciones } \\
\hline 45. Le molestan los efectos secundarios del tratamiento & 0 & 1 & 2 & 3 & 4 \\
\hline 46. Necesita estar acostado & 0 & 1 & 2 & 3 & 4 \\
\hline $\begin{array}{l}\text { 47. Se siente cercano a su pareja } \\
\text { (o a la persona que le da su principal apoyo) }\end{array}$ & 0 & 1 & 2 & 3 & 4 \\
\hline 48. ¿Está satisfecho con su vida sexual? & 0 & 1 & 2 & 3 & 4 \\
\hline 49. ¿Su médico atiende satisfactoriamente sus dudas? & 0 & 1 & 2 & 3 & 4 \\
\hline 50. Está satisfecho de cómo usted está afrontando su enfermedad & 0 & 1 & 2 & 3 & 4 \\
\hline 51. Se siente nervioso & 0 & 1 & 2 & 3 & 4 \\
\hline 52. Le preocupa que su enfermedad empeore & 0 & 1 & 2 & 3 & 4 \\
\hline 53. Duerme bien & 0 & 1 & 2 & 3 & 4 \\
\hline $\begin{array}{l}\text { 54. Siente que empeoran los síntomas de su enfermedad } \\
\text { con el calor }\end{array}$ & 0 & 1 & 2 & 3 & 4 \\
\hline 55. Tiene dificultad para controlar su orina & 0 & 1 & 2 & 3 & 4 \\
\hline 56. Orina con más frecuencia que normalmente & 0 & 1 & 2 & 3 & 4 \\
\hline 57. Le molestan los escalofríos & 0 & 1 & 2 & 3 & 4 \\
\hline 58. Tiene episodios de fiebre que le molestan & 0 & 1 & 2 & 3 & 4 \\
\hline 59. Se siente molesto con los espasmos musculares & 0 & 1 & 2 & 3 & 4 \\
\hline
\end{tabular}

chiatric patient outcome: the wisdom of Gheel confirmed. Arch Gen Psychiatry 1980; 37: 129-32.

23. Andrews FM, Withey SB. Social indicators of well-being: Americans' perceptions of life quality. New York: Plenum; 1976.

24. Krupp L, Álvarez L, LaRocca L, Scheinberg L. Fatigue in multiple sclerosis. Arch Neurol 1988; 45: 435-41.

25. Vickrey BG, Hays RD, Genovese BJ, Myers LW, Ellison GW. Compa- rison of a generic to disease-targeted health-related quality-of-life measures for multiple sclerosis. J Clin Epidemiol 1997; 50: 557-69.

26. Murphy N, Confavreux C, Haas J, König N, Roullet E, Sailer M, et al. Quality of life in multiple sclerosis in France, Germany and the United Kingdom. J Neurol Neurosurg Psychiatry 1998; 65: 460-6.

27. Aronson KJ. Quality of life among persons with multiple sclerosis and their caregivers. Neurology 1997; 48: 74-80.

28. Lankhorst GJ, Jelles F, Smits RCF, Polman CH, Kuik DJ, Pfennings 
L, et al. Quality of life in multiple sclerosis: the disability and impact profile (DIP). J Neurol 1996; 243: 469-74.

29. Gronning M, Hannisdal E, Mellgren S. Multivariate analyses of factors associated with unemployment in people with multiple sclerosis. J Neurol Neurosurg Psychiatry 1990; 53: 388-90.

30. De Andrés C, Guillem A. Una aproximación sobre la calidad de vida en pacientes con esclerosis múltiple. Rev Neurol 2000; 30: 1229-34.

31. Cella DF, Dineen MA, Arnason B, Reder MD, Webster MA, Karabatsos BA, et al. Validation of the Functional Assessment of Multiple Sclerosis quality of life instrument. Neurology 1996; 47: 129-39.

32. Martínez-Yélamos S, Martínez-Yélamos A, Hernández-Regadera JJ, Martín-Ozaeta G, Arbizu-Urdiain T. Impacto sociolaboral de la esclerosis múltiple. Neurologia 1999; 14: 107-10.

33. Asociación de Esclerosis Múltiple de Bizkaia. Estudio psicomédico y sociolaboral sobre personas con esclerosis múltiple. Barcelona: Federación Española para la Lucha contra la Esclerosis Múltiple; 1999.

34. Poser CM, Paty DW, Scheinberg L, McDonald WI, Davis FA, Ebers GC, et al. New diagnostic criteria for multiple sclerosis: guidelines for research protocols. Ann Neurol 1983; 13: 227-31.

35. Weinberger M, Oddone EZ, Samsa GP, Landsman PB. Are health-related quality-of-life measures affected by the mode of administration? J Clin Epidemiol 1996; 49: 135-40.

36. Hamilton M. A rating scale for depression. J Neurol Neurosurg Psychiatry $1960 ; 23: 56-62$.

37. Halmiton M. The development of a scale for primary depressive illness. Br J Soc Clin Psychol 1967; 6: 278-96.

38. Folstein MF, Folstein SE, Mcchugh PR. 'Mini-Mental State'. A practical method for grading the cognitive state of patients for the clinician. J Psychiatr Res 1975; 12: 189-98.

39. Cacho J, García-García R, Arcaya J, Vicente JL, Lantada N. Una propuesta de aplicación y puntuación del test del reloj en la enfermedad de Alzheimer. Rev Neurol 1999; 28: 648-55.

40. Elkins LE, Strober LB, Scherl WF, Perone PS, Demento L, Krupp LB, et al. Brief cognitive screening in multiple sclerosis: MiniMental State Examination and Clock Drawing Test. Neurology 2000; 54: 56.

\section{HACIA LA BÚSQUEDA DE DIMENSIONES MÁS ESPECÍFICAS EN LA MEDICIÓN DE LA CALIDAD DE VIDA EN LA ESCLEROSIS MÚLTIPLE}

Resumen. Introducción y objetivos. El estudio de la calidad de vida en la esclerosis múltiple (EM) debe contemplar el uso de un instrumento que abarque las dimensiones social, psicológica, física y mental. El objetivo de este estudio es mostrar las dimensiones validadas del cuestionario de calidad de vida Functional Assessment of Multiple Sclerosis (FAMS) en la población española, y verificar la consistencia de éstas. Pacientes y métodos. Se seleccionaron 308 pacientes con EM (clínicamente definida o con apoyo de laboratorio, según los criterios clínicos de Poser), procedentes de 10 consultas externas de Neurología pertenecientes a hospitales de la Comunidad Autónoma de Madrid. El diseño del estudio es transversal y se llevó a cabo a través de entrevista directa. Se utilizó un cuestionario que contenía el FAMS y otras variables referentes a diversos aspectos (clínicos, sociales, laborales, psicológicos y cognitivos). Resultados. La edad media es de 38,2 \pm 10,5 años, con una puntuación media en la escala Expanded Disability Status Scale (EDSS) de 3,6 $\pm 2,3$. Las puntuaciones medias de las dimensiones son inferiores a las obtenidas en la versión original del cuestionario FAMS; la consistencia interna de estas dimensiones es levemente más elevada que las del FAMS 'original'. Las dimensiones 'síntomas' y 'estado de ánimo general', así como el FAMS en su conjunto, ven incrementadas su consistencia interna con la inclusión de ocho de las variables que se excluyeron originalmente. Conclusión. El cuestionario FAMS muestra una mayor consistencia y fiabilidad en la evaluación de la calidad de vida, si incluimos variables específicas de la EM que no formaban parte del FAMS en su versión americana. [REV NEUROL 2001; 32: 705-13]

Palabras clave. Calidad de vida. Depresión. Deterioro cognitivo. Esclerosis múltiple. Incapacidad. Movilidad.
41. Iriarte J, Carreño M, De Castro P. Fatiga y afectación por sistemas funcionales en esclerosis múltiple. Neurologia 1996; 11: 210-15.

42. Tola MA, Yugueros MI, Fernández-Buey N, Marco J, Gutierrez-García JM, Gómez-Nieto J, et al. Deficiencia, discapacidad y minusvalía en la esclerosis múltiple: un estudio de base poblacional en Valladolid. Rev Neurol 1998; 26: 728-34.

43. The Canadian Burden of Illness Study Group. Burden of illness of multiple sclerosis. Part II. Quality of life. Can J Neurol Sci 1998; 25: 31-8.

44. Riether AM. Anxiety in patients with multiple sclerosis. Semin Clin Neurospychiatry 1999; 4: 103-13.

45. Pacenham KL. Adjustment to multiple sclerosis: application of a stress and coping model. Health Psychol 1999; 18: 383-92.

46. Patten SB, Metz LM. Depression in multiple sclerosis. Psychother Psychosom 1997; 66: 286-92.

47. Hammond SR, McLeod JG, Macaskill P, English DR. Multiple sclerosis in Australia: socieconomic factors. J Neurol Neurosurg Psychiatry 1996; 61: 311-3.

48. Provinciali J, Ceravolo MG, Bartolini M, Logullo F, Danni M. A multidimensional assessment of multiple sclerosis: relationships between disability domains. Acta Neurol Scand 1999; 100: 156-62.

49. Camp SJ, Stevenson VL, Thompson AJ, Miller DH, Borras C, Auriacombe $\mathrm{S}$, et al. Cognitive function in primary progressive and transitional progressive multiple sclerosis: a controlled study with MRI correlates. Brain 1999; 122: 1341-8.

50. Rao SM, Gary JL, Nauertz BS, Berbardin L, Unverzagt F. Cognitive disfunction in multiple sclerosis. I. Frequency, patterns and prediction. Neurology 1991; 41: 685-91.

51. Rao SM, Leo GJ, Ellington M, Nauertz BS, Bernardin L, Unverzagt F. Cognitive dysfunction in multiple sclerosis. II. Impact on employment and social functioning. Neurology 1991; 41: 692

52. Mohr DC, Goodkin DE, Gatto N, Van der Wende J. Depression, coping and level of neurological impairment in multiple sclerosis. Mult Scler 1997; 3: 254-8.

53. Gilchrist AC, Creed FH. Depression, cognitive impairment and social stress in multiple sclerosis. J Psychosom Res 1994; 38: 193-201.

\section{A BUSCA DE DIMENSÕES MAIS ESPECÍFICAS NA MEDIÇÃO DA QUALIDADE DE VIDA NA ESCLEROSE MÚLTIPLA}

Resumo. Introdução e objectivos. O estudo da qualidade de vida na esclerose múltipla (EM) deve contemplar o uso de um instrumento que englobe as dimensões social, psicológica, física e mental. $O$ objectivo deste estudo é mostrar as dimensões validadas da escala de qualidade de vida Functional Assessment of Multiple Sclerosis (FAMS) na população espanhola, e verificar a consistência das mesmas. Doentes e métodos. 308 doentes com EM (clinicamente definida ou através do apoio de exames laboratoriais segundo os critérios clínicos de Poser), provenientes de 10 consultas externas de Neurologia pertencentes a Hospitais da Comunidade Autónoma de Madrid. O desenho do estudo é transversal e foi realizado através de entrevista directa. Foi utilizado um questionário que incluia a escala FAMS e outras variáveis referentes a diversos aspectos (clínicos, sociais, laborais, psicológicos e cognitivos). Resultados. A idade média é de 38,2 \pm 10,5 anos com uma pontuação média na escala Expanded Disability Status Scale (EDSS) de 3,6 $\pm 2,3$. As pontuações médias das dimensões são inferiores às obtidas na versão original do FAMS; a consistência interna destas dimensões é ligeiramente mais elevada que as do FAMS 'original'. As dimensões 'sintomas' $e$ 'estado de ânimo geral', assim como o FAMS no seu conjunto, são complementadas pela inclusão de oito das variáveis que foram excluídas originalmente. Conclusão. A escala FAMS mostra maior consistência e fiabilidade na avaliação da qualidade de vida, se incluirmos variáveis específicas da EM que não faziam parte do FAMS na sua versão americana. [REV NEUROL 2001; 32: 705-13]

Palavras chave. Depressão. Deterioração cognitiva. Esclerose múltipla. Incapacidade. Mobilidade. Qualidade de vida. 